\title{
Citrate Synthase Gene Comparison, a New Tool for Phylogenetic Analysis, and Its Application for the Rickettsiae $\dagger$

\author{
VERONIQUE ROUX, ${ }^{1}$ ELENA RYDKINA, ${ }^{1,2}$ MARINA EREMEEVA, ${ }^{1}$ AND DIDIER RAOULT ${ }^{1 *}$ \\ Unité des Rickettsies, Centre National de la Recherche Scientifique, EPJ 0054, Faculté de Médecine, \\ 13385 Marseille, France, ${ }^{1}$ and The Gamaleya Research Institute of Epidemiology and \\ Microbiology, Russian Academy of Medical Sciences, 123098 Moscow, Russia ${ }^{2}$
}

\begin{abstract}
Using PCR and an automated laser fluorescent DNA sequencer, we amplified and sequenced a 1,234-bp fragment of the citrate synthase-encoding gene ( $g l t A$ ) of 28 bacteria belonging to the genus Rickettsia. Comparative sequence analysis showed that most of the spotted fever group (SFG) rickettsiae belonged to one of two subgroups. The first subgroup included Rickettsia massiliae, strain Bar 29, Rickettsia rhipicephali, "Rickettsia aeschlimanni," and Rickettsia montana, which have been isolated only from ticks. The second subgroup was larger and included the majority of the human pathogens and also rickettsiae isolated only from ticks; the members of this subgroup were strain S, Rickettsia africae, "Rickettsia mongolotimonae," Rickettsia sibirica, Rickettsia parkeri, Rickettsia conorii, Rickettsia rickettsii, the Thai tick typhus rickettsia, the Israeli tick typhus rickettsia, the Astrakhan fever rickettsia, "Rickettsia slovaca," and Rickettsia japonica. The sequence analysis also showed that the tick-borne organisms Rickettsia helvetica and Rickettsia australis and the mite-borne organism Rickettsia akari were associated with the SFG cluster; that Rickettsia prowazekii and Rickettsia typhi, two representatives of the typhus group, clustered together; and that Rickettsia canada, Rickettsia bellii, and the AB bacterium probably represent three new groups. We compared the phylogenetic trees inferred from citrate synthase gene sequences and from 16S ribosomal DNA (rDNA) sequences. For rickettsial phylogeny, the citrate synthase approach was more suitable, as demonstrated by significant bootstrap values for all of the nodes except those in the larger subgroup defined above. We also compared phylogenetic analysis results obtained in a comparison of the sequences of both genes for all of the representatives of the domain Bacteria for which the glt $A$ sequence was determined. We believe that comparison of gltA sequences could be a complementary approach to $16 \mathrm{~S}$ rDNA sequencing for inferring bacterial evolution, especially when unstable phylogenetic models are obtained from ribosomal sequences because of high levels of sequence similarity between the bacteria studied.
\end{abstract}

Rickettsiae belong to the order Rickettsiales, whose members were described as obligately intracellular gram-negative microorganisms (55). Several species cause disease in humans or in other vertebrate and invertebrate hosts and have a worldwide distribution. Recently, on the basis of 16S rRNA sequence similarity, Rickettsia tsutsugamushi was removed from the genus Rickettsia and was renamed Orientia tsutsugamushi (48). The genus Rickettsia is presently divided into two groups: the typhus group (TG), which includes three species (Rickettsia prowazekii, the agent of the epidemic typhus, Rickettsia typhi, which causes murine typhus, and Rickettsia canada, which has been isolated only from ticks), and the spotted fever group (SFG). The number of recognized members of the SFG increased following the development of an improved cell culture isolation technique (the shell vial technique) (26) and an increased interest in arthropod-transmitted diseases. New SFG rickettsiae have been isolated worldwide from arthropods; these organisms include Rickettsia massiliae, which was isolated in France (6) and in Greece (3), "Rickettsia mongolotimonae," which was isolated in the People's Republic of China (63), strain Bar 29, which was isolated in Spain (7), "Rickettsia aeschlimanni," which was isolated in Morocco (5), Rickettsia hel-

\footnotetext{
* Corresponding author. Mailing address: Unité des Rickettsies, Faculté de Médecine, Centre National de la Recherche Scientifique EPJ 0054, 27 Boulevard Jean Moulin, 13385 Marseille, France. Phone: (33) 04-91-38-55-17. Fax: (33) 04-91-83-03-90. E-mail: RAOULT @pacwan.mm-soft.fr.

$\dagger$ This work is dedicated to Natalia Balayeva, who participated in the beginning of the study and died during the time of this work.
}

vetica, which was isolated in Switzerland (11), and the strain $\mathrm{AB}$ bacterium, which was isolated in England (57) (this strain has been characterized by genomic criteria but it remains unisolated). In addition, new SFG rickettsiae that have been isolated from humans include Rickettsia africae (from Zimbabwe) (24), Rickettsia japonica (from Japan) (49), Rickettsia felis (from the United States) $(22,44)$, the Astrakhan fever rickettsia (from Russia) (17), and "Rickettsia mongolotimonae" (from France) (37). These organisms were characterized by using phenotypic methods (serotyping and protein analysis by sodium dodecyl sulfate-polyacrylamide gel electrophoresis) (1) and genomic methods (restriction fragment length polymorphism [RFLP] analysis of PCR amplification products [18, 38], pulsed-field gel electrophoresis [40], and 16S rRNA sequencing $[41,45])$. Several studies aimed at assessing the genotypic relationships of rickettsiae have been performed by using randomly chosen DNA probes and RFLP analysis (36), PCRRFLP profiling of the genes encoding citrate synthase (glt $A)$ and protein rOmpA (a high-molecular-weight outer membrane protein) (38), or macrorestriction analysis with pulsedfield gel electrophoresis (40). These methods were useful for rickettsial identification and differentiation but were not really suitable for phylogenetic study. The most widely used approach for phylogenetic study of bacteria is based on comparisons of 16S rRNA gene sequences (60). This approach has already been used to demonstrate that the genus Rickettsia belongs to group 1 of the $\alpha$ subclass of the Proteobacteria and has a common origin with the genera Ehrlichia, Cowdria, and Anaplasma and Wolbachia pipientis (54). A high level of conservation in the sequence of this gene has been demonstrated 
for the members of the genus Rickettsia (levels of similarity between two species, more than $97.2 \%$ ); thus, reliable phylogenetic analysis based on a comparison of the $16 \mathrm{~S}$ rRNA gene sequences has been difficult, especially for the members of the SFG.

The importance of comparing results obtained for multiple molecules to test the reliability of organismal phylogeny has recently been highlighted, and it has been suggested that any alternative genes used should be genes whose products perform central cellular functions (32). At present, few genes have been sequenced in rickettsial genomes. We chose glt $A$ as a possible tool for assessing more precise phylogenetic relationships among the rickettsiae. Citrate synthase is a component of nearly all living cells and is an enzyme of a central metabolic pathway, the citric acid cycle, which plays a key role in energy production and in providing important biosynthetic precursors (58). Two main types of citrate synthase have been identified, and these two types are made up of identical subunits that have $M_{\mathrm{r}} \mathrm{s}$ of approximately 50,000 ; a dimeric form is found in members of the Eukarya and gram-positive bacteria, and a multimeric form (with four to six identical subunits) is found in members of the Proteobacteria. The two forms of the enzyme differ in their regulatory sensitivities; the dimer is sensitive in vitro to inhibition by ATP, whereas the multimeric form is inhibited by $\mathrm{NADH}$ and 2-oxoglutarate. The amino acid sequences of citrate synthase either have been determined directly from the protein or have been inferred from DNA sequences for members of the domain Eukarya (including pig [9], Tetrahymena thermophila [30], Arabidopsis thaliana [50], and Saccharomyces cerevisiae [46], for members of the domain Bacteria (including Escherichia coli [28], Acinetobacter anitratum [14], R. prowazekii [62], Coxiella burnetii [21], Bartonella henselae [29], Pseudomonas aeruginosa [15], Conynebacterium glutamicum [16], Bacillus coagulans [43], and Mycobacterium smegmatis [12]), and for a representative of the domain $A r-$ chaea, Thermoplasma acidophilum (47). Multiple alignments of these protein sequences have revealed significant levels of similarity between intradomain Eukarya sequences (46 to 92\%) and between intraphylum Proteobacteria sequences (56 to $75 \%$ ); however, the interdomain levels of similarity are far lower (20 to $26 \%$ ) (47). It appears, therefore, that the citrate synthase gene may be sufficiently informative to analyze the evolutionary relationships of closely related bacteria.

It has been determined that the rickettsiae can accumulate ATP directly from the cytoplasm of the host cell. However, these organisms are not strict energy parasites and can generate their own ATP via the tricarboxylic acid cycle and oxydative phosphorylation (59). Despite the resemblance of the morphology and cell wall chemistry of rickettsiae to the morphology and cell wall chemistry of typical gram-negative bacteria, the citrate synthase of $R$. prowazekii has been shown to resemble to the enzymes of members of the Eukarya and grampositive bacteria in terms of its structure and regulation properties (33), but a sequence comparison has shown that it is most closely related to the enzymes of gram-negative bacteria. The glt $A$ gene was the first rickettsial gene that was cloned and sequenced, but only one entire sequence is available, that of $R$. prowazekii (62). However, it was shown by PCR amplification that this gene was present on the chromosomes of all rickettsiae $(18,38)$. We amplified and sequenced the glt $A$ gene of 28 rickettsiae and used alignments of the sequences as the basis for a phylogenetic analysis. The results obtained were compared with the results obtained when phylogenic conclusions were inferred from $16 \mathrm{~S}$ ribosomal DNA (rDNA) sequences.

\section{MATERIALS AND METHODS}

Rickettsial strains. All of the rickettsial strains included in this study are listed in Table 1.

(i) Validly published species. The following four strains were obtained from the American Type Culture Collection: Rickettsia conorii Moroccan and Seven (= Malish), Rickettsia rickettsii R (= Bitteroot), and Rickettsia akari MK (= Kaplan). Rickettsia rhipicephali 3-7-6 R. africae ESF-5, and Rickettsia australis Phillips were supplied by G. A. Dasch, Naval Medical Research Institute, Bethesda, Md. The following four organisms were a gift from D. H. Walker, University of Texas, Galveston: R. japonica YM, Rickettsia parkeri Maculatum 20, Rickettsia montana M/5-6, and Rickettsia bellii. Two strains, $R$. massiliae Mtu1 and GS, were isolated in our laboratory from ticks found in France and in Greece, respectively. Rickettsia sibirica $246, R$. prowazekii Breinl, $R$. typhi Wilmington, and $R$. canada 2678 were obtained from the Gamaleya Research Institute Collection. An isolate of $R$. helvetica was provided by W. Burgdorfer, Rocky Mountain Laboratory, Hamilton, Mont. R. conorii M1 and Indian tick typhus rickettsia were obtained from the Gamaleya Research Institute Collection.

(ii) Species which have not been validly published. The following three strains were a gift from G. A. Dasch: "Rickettsia slovaca" 13-B, Thai tick typhus rickettsia strain TT-118, and Israeli tick typhus rickettsia strain ISTT CDC1. Strain $S$ was obtained from the Gamaleya Research Institute Collection. Ladybird beetles infected with the $\mathrm{AB}$ bacterium were provided by I. Zakharov, Vavilov Institute of General Genetics, Russian Academy of Science, Moscow. " $R$. mon golotimonae" HA-91 and Astrakhan fever rickettsia strain A-167 were isolated in our laboratory from ticks collected in the Alashian region of the Inner Mongolia autonomous region and in the Astrakhan region of Russia, respectively. Strain Bar 29 and " $R$. aeschlimanni" MC16 were isolated in our laboratory from ticks collected in Spain and Morocco, respectively.

Rickettsial cultivation and nucleic acid preparation. All strains were propagated on Vero cell monolayers (catalog no. CRL 1587; American Type Culture Collection). Dulbecco modified minimal essential medium (Eurobio, Les Ulis, France) supplemented with $10 \%$ fetal calf serum (Eurobio) and $2 \%$ L-glutamine (Eurobio) was used for cultivation of $R$. prowazekii, minimal essential medium (MEM) (Eurobio) supplemented with $2 \%$ fetal calf serum and $2 \%$ L-glutamine was used for cultivation of $R$. typhi, and MEM supplemented with $4 \%$ fetal calf serum and $1 \% \mathrm{~L}$-glutamine was used for cultivation of all of the other rickettsiae. Infected cells were maintained in a $5 \% \mathrm{CO}_{2}$ atmosphere at $35^{\circ} \mathrm{C}$ for $R$. prowazekii and $R$. typhi and at $32^{\circ} \mathrm{C}$ for all of the other rickettsiae. Infection of Vero cells was monitored by Gimenez staining, and rickettsiae were harvested at the optimal level of multiplication (after 3 to 5 days). Rickettsial cultures were centrifuged at $12,000 \times g$ for $10 \mathrm{~min}$, resuspended in Dulbecco modified minimal essentia medium or MEM, and stored at $-70^{\circ} \mathrm{C}$.

Genomic DNA extracts were prepared as described previously (38). AB bacterium DNA was extracted from a ladybird beetle by using Qiagen columns (QIAamp tissue kit; QIAGEN, Hilden, Germany).

PCR amplification and sequencing method. PCR amplification and sequencing reactions were performed by using the oligonucleotide primers listed in Table 2. Oligonucleotides were purchased from Eurogentec S.A., Seraing, Belgium. Each PCR was carried in a Peltier model PTC-200 thermal cycler (MJ Research, Inc., Watertown, Mass.). AmpliTaq DNA polymerase and solutions of $\mathrm{MgCl}_{2}$ and PCR buffer II were purchased from Perkin-Elmer Cetus, Norwalk, Conn. and the deoxynucleoside triphosphate solutions were purchased from Boehringer Mannheim Biochemicals, Indianapolis, Ind.

A 380-bp fragment of the glt $A$ gene was amplified by using primers Rp $877 \mathrm{p}$ and $\mathrm{Rp} 1258 \mathrm{n}$ as described by Regnery et al. (38). The $5^{\prime}$-terminal region and the 3 '-terminal region of the citrate synthase gene were PCR amplified by using primers CS1d (or CSM18d for $R$. akari and R. australis) and CS890r and primers Rp877p and CS1273r, respectively.

Primers CS1d, CSM18d, and CS1273r were chosen by referring to the glt $A$ sequence of $R$. prowazekii (62), and CS890r was selected following recognition of a conserved region within the gene after alignment of the sequences of the PCR fragments amplified with primers Rp877p and Rp1258n for all of the rickettsiae included in the study. The sequencing primers used were the primers used for PCR amplification, direct primers CS113d, CS113Bd, CS409d, CS535d, and CS1048d, and reverse primers CS244r, CS428r, CS715r. These primers were complementary to gltA conserved regions, as determined by alignment of the sequences. The sequencing primers were labeled in the $5^{\prime}$-terminal region with fluorescein isothiocyanate during oligonucleotide synthesis (Eurogentec).

A 3- $\mu$ l portion of rickettsial genomic DNA was amplified in a 100- $\mu$ l reaction mixture containing $10 \mathrm{pmol}$ of each primer, $200 \mu \mathrm{M}$ dATP, $200 \mu \mathrm{M}$ dCTP, 200 $\mu \mathrm{M}$ dGTP, $200 \mu \mathrm{M}$ dTTP, $1.25 \mathrm{U}$ of AmpliTaq DNA polymerase, and $0.2 \mu \mathrm{M}$ $\mathrm{MgCl}_{2}$ in $1 \times \mathrm{PCR}$ buffer II. Amplification was carried under the following conditions: an initial 2-min denaturation step at $95^{\circ} \mathrm{C}$ was followed by 40 cycles consisting of denaturation at $95^{\circ} \mathrm{C}$ for $30 \mathrm{~s}$, annealing at $45^{\circ} \mathrm{C}$ for $30 \mathrm{~s}$, and extension at $65^{\circ} \mathrm{C}$ for $55 \mathrm{~s}$. Amplification was completed by incubation for $3 \mathrm{~min}$ at $72^{\circ} \mathrm{C}$ to allow complete extension of the PCR products. To verify the results of amplification, $10 \mu \mathrm{l}$ of the PCR product was resolved at $8 \mathrm{~V} / \mathrm{cm}$ in a $1 \%$ agarose gel (type LE; Sigma-Aldrich Chimie, St. Quentin Fallavier, France) by using $1 \times$ TBE migration buffer $(0.089 \mathrm{M}$ Tris base, $0.089 \mathrm{M}$ boric acid, $0.002 \mathrm{M}$ EDTA; $\mathrm{pH} 8.0$ ). The size of the PCR product was determined by comparison 
TABLE 1. Rickettsial strains studied

\begin{tabular}{|c|c|c|c|c|}
\hline Taxon & Strain & Source & Geographic origin & Human disease \\
\hline R. conorii & Moroccan & Unknown & Morocco & Mediteranean spotted fever \\
\hline R. conorii & Seven (= Malish) & Unknown & South Africa & Mediteranean spotted fever \\
\hline R. conorii & M1 & Rhipicephalus sanguineus & Georgia (formerly USSR) & \\
\hline R. conorii & $\begin{array}{l}\text { Indian tick typhus } \\
\text { rickettsia }\end{array}$ & Rhipicephalus sanguineus & India & \\
\hline Astrakhan fever rickettsia & A-167 & Rhipicephalus pumilio & $\begin{array}{l}\text { Astrakhan region (formerly } \\
\text { USSR) }\end{array}$ & Astrakhan fever \\
\hline Israeli tick typhus rickettsia & ISTT CDC1 & Human & Israel & Israeli spotted fever \\
\hline R. sibirica & 246 & Dermacentor nuttali & Former USSR & Siberian tick typhus \\
\hline "R. mongolotimonae" & HA-91 & Haemophysalis asiaticum & Inner Mongolia & Unnamed spotted fever \\
\hline R. parkeri & Maculatum 20 & Amblyomma maculatum & Mississippi & \\
\hline R. africae & ESF-5 & Amblyomma variegatum & Shulu Province, Ethiopia & African tick bite fever \\
\hline Strain S & S & Rhipicephalus sanguineus & Armenia (formerly USSR) & \\
\hline "R. slovaca" & $13-\mathrm{B}$ & Dermacentor marginatus & Slovakia & \\
\hline Thai tick typhus rickettsia & TT-118 & Ixodes sp. or Rhipicephalus sp. & Thailand & \\
\hline R. rickettsii & $\mathrm{R}(=$ Bitterroot $)$ & Dermacentor andersoni & Montana & Rocky Mountain spotted fever \\
\hline R. japonica & YM & Human & Japan & Oriental spotted fever \\
\hline R. massiliae & Mtu 1 & Rhipicephalus turanicus & Camargue, France & \\
\hline R. massiliae & GS & Rhipicephalus sanguineus & Greece & \\
\hline Bar 29 & Bar 29 & Rhipicephalus sanguineus & Spain & \\
\hline R. rhipicephali & $3-7-6$ & Rhipicephalus sanguineus & Mississippi & \\
\hline "R. aeschlimanni" & MC16 & Hyaloma marginatum & Morocco & \\
\hline R. montana & $\mathrm{M} / 5-6$ & Microtus sp. & Montana & \\
\hline R. australis & Phillips & Human & Queensland, Australia & Queensland tick typhus \\
\hline R. akari & MK (= Kaplan $)$ & Human & New York, N.Y. & Rickettsial pox \\
\hline R. helvetica & $\mathrm{C} 9 \mathrm{P9}$ & Ixodes ricinus & Switzerland & \\
\hline R. bellii & $369 \mathrm{~L} 42-1$ & Dermacentor variabilis & Ohio & \\
\hline R. canada & 2678 & Haemophysalis leporispalustris & Canada & \\
\hline $\mathrm{AB}$ bacterium & & Adalia bipunctata & England & \\
\hline R. prowazekii & Brein L & Human & Poland & Epidemic typhus \\
\hline R. typhi & Wilmington & Human & North Carolina & Murine typhus \\
\hline
\end{tabular}

with DNA molecular weight marker VI (Boehringer Mannheim). PCR products were purified by using a QIAquick Spin PCR purification Kit (QIAGEN). Sequencing reactions were carried out by using an AmpliCycle sequencing kit (Perkin-Elmer Corp., Foster City, Calif.) according to the instructions of the supplier. The reactions were performed at the optimal temperatures $(T)$, as calculated for each primer by using the following formula: $T=[4(n \mathrm{G}+n \mathrm{C})+$ $2(n \mathrm{~A}+n \mathrm{~T})]-5$, where $n$ is the number of bases in the primer. Reactions were stopped by adding $4 \mu \mathrm{l}$ of stop solution (100\% deionized formamide, $6 \mathrm{mg}$ of dextran blue per $\mathrm{ml}, 20 \mathrm{mM}$ EDTA; $\mathrm{pH} 8.3$ ). The products of sequencing reactions were resolved on $6 \%$ denaturing polyacrylamide gels (Pharmacia, St. Quentin-Yveline, France) by using $1 \times$ TBE buffer, and electrophoresis was performed with a model A.L. F. DNA sequencer (Pharmacia LKB). At least two sequencing reactions were performed for each strand of DNA.
Data analysis. The gltA sequences were aligned by using the multisequence alignment program CLUSTAL in the BISANCE software package (13). Phylogenetic relationships were inferred by using version 3.4 of the PHYLIP software package (19). Evolutionary distance matrices, generated by DNADIST, were constructed by the method of Jukes and Cantor (23) and Kimura (25). The matrices were used to infer dendrograms by using the neighbor-joining method (42). The data were also examined by using parsimony analysis (DNAPARS in PHYLIP). A bootstrap analysis was performed to investigate the stability of the trees obtained. Bootstrap values were obtained for a consensus tree based on 100 randomly generated trees by using SEQBOOT and CONSENSE in the same package.

gltA and $16 \mathrm{~S}$ rRNA sequences of $E$. coli, Bartonella henselae, Bacillus coagulans, M. smegmatis, P. aeruginosa, Corynebacterium glutamicum, and Thermo

TABLE 2. Oligonucleotide primers used for PCR amplification and sequencing of rickettsial species

\begin{tabular}{|c|c|c|c|}
\hline Primer & Nucleotide sequence $\left(5^{\prime}-3^{\prime}\right)$ & $\begin{array}{l}\text { Citrate synthase gene } \\
\text { positions relative to the } \\
\text { open reading frame }\end{array}$ & Organisms used in \\
\hline $\mathrm{CS} 1 \mathrm{~d}^{a}$ & ATGACTAATGGCAATAATAA & $1-20$ & All except $R$. australis and $R$. akari \\
\hline $\operatorname{CSM} 18 \mathrm{~d}^{a}$ & GAATGAGGTATATACATTATG & $-18-3$ & $R$. australis, $R$. akari \\
\hline CS890 $\mathrm{r}^{a, b}$ & GCTTTAGCTACATATTTAGG & $890-871$ & All \\
\hline $\mathrm{Rp} 877 \mathrm{p}^{a, b}$ & GGGGACCTGCTCACGGCGG & $797-815$ & All \\
\hline $\mathrm{Rp} 1258 \mathrm{n}^{a, b}$ & ATTGCAAAAAGTACAGTGAACA & $1178-1157$ & All \\
\hline CS1273r $\mathrm{r}^{a, b}$ & CATAACCAGTGTAAAGCTG & $1273-1255$ & All \\
\hline $\operatorname{CS} 113 \mathrm{~d}^{b}$ & GTAGGGTATCTGCGGAAGC & $113-131$ & All except $R$. bellii \\
\hline $\mathrm{CS} 113 \mathrm{Bd}^{b}$ & GTAAAGTATATTCTGAAGCG & $113-132$ & R. bellii \\
\hline $\mathrm{CS} 409 \mathrm{~d}^{b}$ & CCTATGGCTATTATGCTTGC & $409-428$ & All \\
\hline $\mathrm{CS} 535 \mathrm{~d}^{b}$ & GCAATGTCTTATAAATATTC & $535-554$ & All \\
\hline CS1048 $\mathrm{d}^{b}$ & CTTGAAGCTCTCCGCTCTTAA & $1048-1067$ & All \\
\hline $\mathrm{CS} 244 r^{b}$ & CTTTAATATCATATCCTCGAT & $244-224$ & All except $R$. bellii \\
\hline $\mathrm{CS} 428 \mathrm{r}^{b}$ & GCAAGCATAATAGCCATAGG & $428-409$ & All \\
\hline $\mathrm{CS} 715 \mathrm{r}^{b}$ & CATTTTGCTCATGATCAGC & $715-695$ & R. bellii \\
\hline
\end{tabular}

\footnotetext{
${ }^{a}$ Oligonucleotide primer used for PCR amplification.
}

${ }^{b} 5^{\prime}$-Isotiocyanate-labeled primer used for sequencing. 
plasma acidophilum and $16 \mathrm{~S}$ rRNA sequences of the rickettsiae studied were obtained from the GenBank database. The same programs and methods as those described above were used to obtain phylogenetic inferences based on these sequences.

Levels of similarity between gltA sequences and between citrate synthase amino acid sequences were determined by using the homology search functions of DNASIS (Hitachi Software Engineering America, Ltd., Brisbane, Calif.). The glt $A$ sequences were translated into protein sequences by using PCgene software. Nucleotide sequence accession numbers. The glt $A$ sequences of the rickettsiae studied have been deposited in the GenBank database under the following accession numbers: $R$. parkeri, U59732; $R$. sibirica, U59734; " $R$. mongolotimonae, U59731; strain S, U59735; $R$ africae, U59733; $R$ conorii, U59730; $R$ rickettsii, U59729; Astrakhan fever rickettsia, U59728; Israeli tick typhus rickettsia, U59727; Thai tick typhus rickettsia, U59726; " $R$. slovaca," U59725; $R$. japonica, U59724; $R$. montana, U74756; $R$. massiliae, U59719; strain Bar 29 $\mathrm{U} 59720 ; R$. rhipicephali, U59721; " $R$. aeschlimanni," U59722; $R$. helvetica, U59723; $R$. australis, U59718; $R$. akari, U59717; $R$. typhi, U59714; AB bacterium, $\mathrm{U} 59712 ; R$. canada, U59713; and $R$. bellii, U59716.

\section{RESULTS}

PCR amplification of rickettsial DNA and determination of the rickettsial gltA sequence. PCR amplification of the 5'terminal region of the citrate synthase gene was obtained with primers CS1d and CS890r for all of the strains studied except the $R$. akari and $R$. australis strains. For these two strains, amplification of the 5'-terminal region of the gene was achieved by using an alternative primer for the direct strand of DNA, primer CSM18d, which localized 18 nucleotides upstream from the first base of the open reading frame. Subsequent sequencing demonstrated five and nine mutations in the region complementary to primer CS1d in $R$. akari and $R$. australis, respectively, which explained the lack of PCR amplification with primer CS1d for these two strains. The middle region of $g l t A$ was amplified for all of the strains studied with primers Rp877n and Rp1258p as described by Regnery et al. (38), and the 3 '-terminal region of the gene was amplified for all of the strains with primers Rp877n and CS1273r. In all, the glt $A$ sequences of three TG isolates and 25 SFG isolates were obtained. The nucleotide sequences determined were between the 21st nucleotide of the designated open reading frame and the 54th nucleotide upstream from the stop codon according to the sequence data for the $R$. prowazekii citrate synthase gene (62). The sequences were 1,234 nucleotides long for all of the samples studied except the $R$. rickettsii and $R$. bellii samples. $R$. rickettsii had a deletion of three bases at position 1006 (nucleotide positions are relative to the previously published sequence for the entire gene of $R$. prowazekii), and $R$. bellii had an insertion of three bases at position 455 . The sequence data determined included data for about $89 \%$ of the open reading frame.

The sequences of four isolates of $R$. conorii (M1, Indian tick typhus rickettsia, Moroccan, and Seven) were identical. There was also no difference in the sequences of two isolates of $R$. massiliae (GS and Mtu1).

The levels of DNA sequence similarity between pairs of strains are shown in Table 3. Most of the strains exhibited between 98.5 and $99.9 \%$ similarity; this group included $R$. conorii, the Astrakhan fever rickettsia, the Israeli tick typhus rickettsia, R. sibirica, "R. mongolotimonae," R. parkeri, strain S, $R$. africae, $R$. rickettsii, " $R$. slovaca," the Thai tick typhus rickettsia, $R$. japonica, $R$. massiliae, strain Bar 29, R. rhipicephali, " $R$. aeschlimanni," and $R$. montana. However, the levels of similarity between representatives of this group and three other members of the SFG rickettsiae ( $R$. helvetica, $R$. akari, and $R$. australis) were lower, ranging from 93.7 to $96.9 \%$.

The level of similarity between $R$. prowazekii and $R$. typhi, two members of the TG, was $96.5 \%$.

The levels of sequence similarity of $R$. canada and the AB bacterium with representatives of the SFG rickettsiae and the TG rickettsiae were 90 to 93 and 89 to $90 \%$, respectively.

$R$. bellii exhibited the lowest levels of sequence similarity $(86.2$ to $86.7 \%$ ) with most other representatives of the SFG the two exceptions were $R$. akari (level of similarity, 95.5\%) and $R$. australis $(95.8 \%)$. R. bellii also exhibited low levels of similarity with members of the TG rickettsiae ( 85 to $85.5 \%$ ).

Similarities among the bacterial glt $A$ sequences available in the GenBank database and among the corresponding amino acid sequences. Similarity data are shown in Table 4 . The levels of glt $A$ sequence similarity within the Proteobacteria and within the gram-positive bacteria were between 56.3 and $68.4 \%$ (between 56.7 and $75 \%$ for proteinic sequences [PS]) and between 46.1 and $47.1 \%$ (between 29.2 and $34.3 \%$ for PS), respectively. The levels of sequence similarity between members of the Proteobacteria and the gram-positive bacteria were between 44.8 and $60.5 \%$ (between 24.3 and $51.2 \%$ for PS). The levels of similarity for the member of the Archaea, Thermoplasma acidophilum, and representatives of the domain Bacteria were between 44.5 and $51 \%$ (between 25.7 and $31.2 \%$ for PS).

Phylogenetic analysis. Dendrograms were inferred by using neighbor-joining (Fig. 1) and parsimony methods. Analyses of the glt $A$ sequence data by these two methods yielded slightly different trees (the architectures of the subgroup including strain $\mathrm{S}, R$. africae, $R$. parkeri, $R$. sibirica, and " $R$. mongolotimonae" were different).

At this time, $R$. prowazekii, $R$. typhi, and $R$. canada are classified in the TG. All of the other rickettsiae are placed in the SFG. The phylogenetic analysis based on the glt $A$ sequence comparison showed that $R$. bellii, $R$. canada, and the $\mathrm{AB}$ bacterium are on a branch which diverged before the split of the SFG and TG species. $R$. canada is outside the TG rickettsial cluster made up of $R$. typhi and $R$. prowazekii. $R$. bellii and the $\mathrm{AB}$ bacterium are outside the SFG cluster. $R$. australis and $R$. akari clustered together. $R$. helvetica was found on a distinct branch between the $R$. akari-R. australis cluster and the other SFG rickettsiae. All of the other SFG rickettsiae were unified into two subgroups, one consisting of five organisms (" $R$. aeschlimanni," strain Bar 29, $R$. massiliae, $R$. rhipicephali, and $R$. montana) and the other consisting of the 12 remaining organisms.

A bootstrap analysis of the data was performed to measure the reliability of the branching order proposed by each analysis. A consensus tree derived from 100 trees generated by the neighbor-joining and parsimony methods had exactly the same structure as the tree shown in Fig. 1. The values for the nodes were greater than $82 \%$ for all of the clusters except the large cluster of SFG made up of 12 strains (bootstrap values, 17 to $78 \%$ ).

We compared the trees constructed on the basis of the nucleotide sequences of glt $A$ and the nucleotide sequences of the 16S rRNA-encoding gene of rickettsiae. A bootstrap analysis of the 16S rDNA-derived trees revealed a lack of statistical significance for most of the proposed branching orders.

We compared the results of phylogenetic analyses obtained by comparing the sequences of both the citrate synthase and 16S rRNA-encoding genes for all representatives of the domain Bacteria for which citrate synthase gene data were determined. The results are summarized in Fig. 2. Thermoplasma acidophilum, a member of the domain Archaea, was used as the outgroup. Analysis of the gltA sequence data by neighborjoining and parsimony methods yielded the same tree. The dendrograms inferred from 16S rDNA sequences were slightly different when the two methods were used. Phylogenetic results inferred from the comparisons of glt $A$ and 16S rDNA sequences clustered together the representatives of the $\alpha$ sub- 


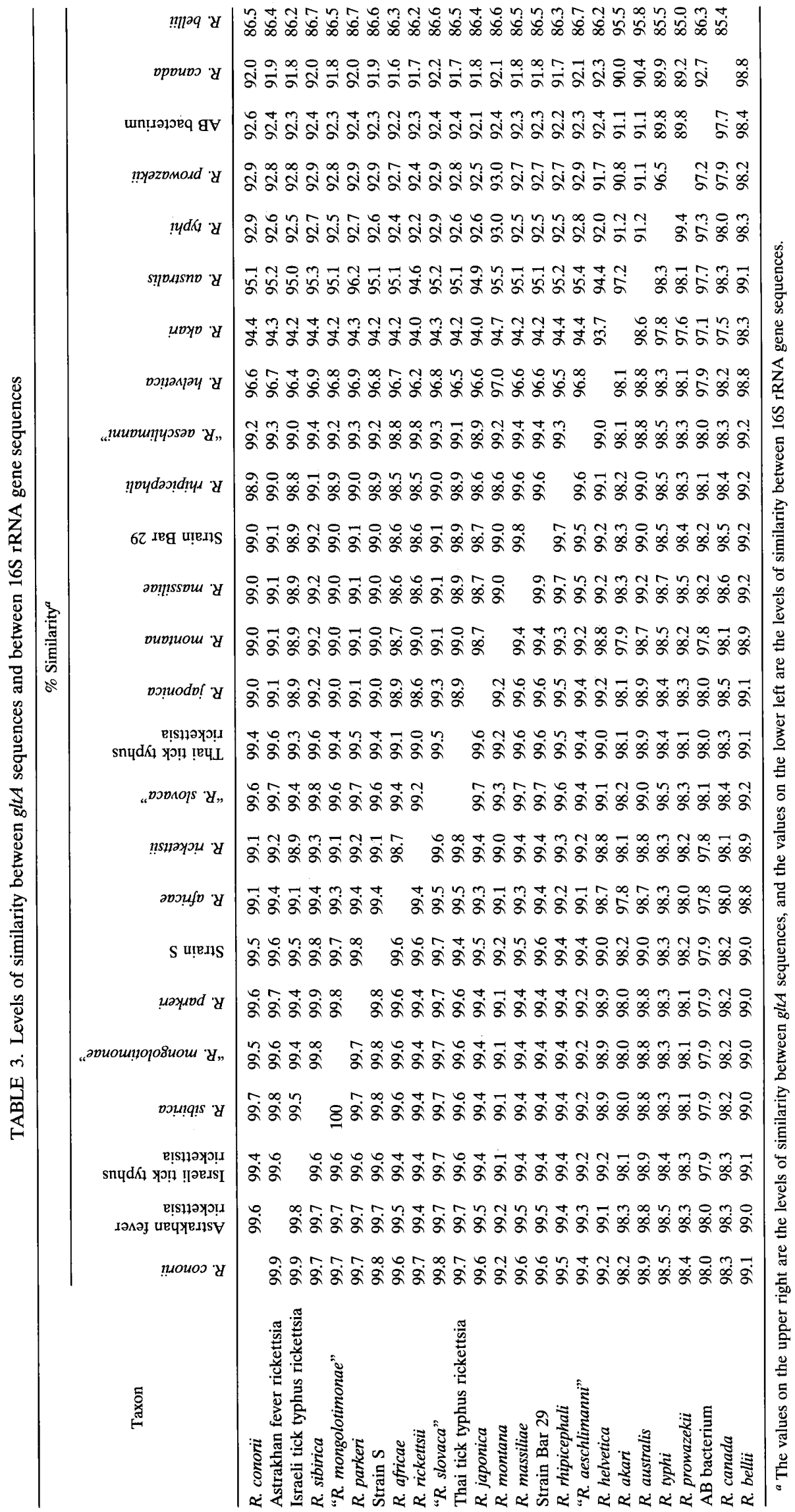


TABLE 4. Levels of similarity between gltA sequences, between citrate synthase amino acid sequences, and between $16 \mathrm{~S}$ rDNA sequences

\begin{tabular}{|c|c|c|c|c|c|c|c|c|c|c|}
\hline \multirow[b]{2}{*}{ Species } & \multicolumn{10}{|c|}{$\%$ Similarity ${ }^{a}$} \\
\hline & 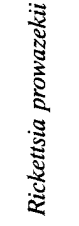 & 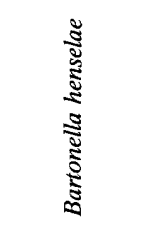 & 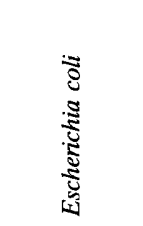 & 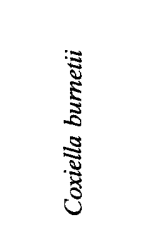 & 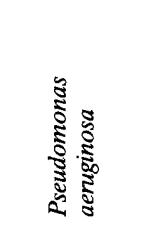 & 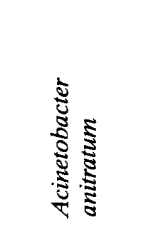 & 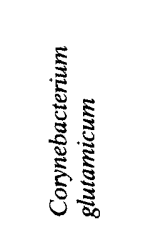 & 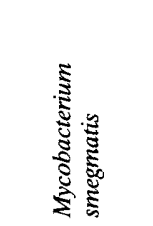 & 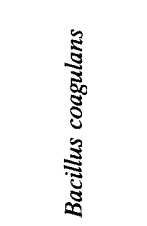 & 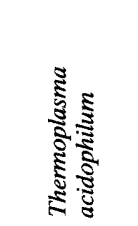 \\
\hline Rickettsia prowazekii & 100 & $65.2(60.3)$ & $61.4(58.9)$ & $61.5(56.7)$ & $56.3(59.4)$ & $62.6(60.3)$ & $51.2(48.9)$ & $44.5(29.4)$ & $48.7(26.5)$ & $48.1(29.9)$ \\
\hline Bartonella henselae & 83.8 & 100 & $65.9(69.3)$ & $63.3(62.1)$ & $60.5(65.4)$ & $65.4(65.6)$ & $52.6(48.7)$ & $45.8(28.1)$ & $48.0(29.8)$ & $44.5(28.6)$ \\
\hline Escherichia coli & 68.5 & 69.4 & 100 & $63.6(61.3)$ & $68.4(69.0)$ & $67.0(67.0)$ & $57.8(49.1)$ & $44.1(24.6)$ & $45.1(29.5)$ & $47.9(25.7)$ \\
\hline Coxiella burnetii & 68.1 & 71.2 & 83.0 & 100 & $62.8(66.6)$ & $64.0(64.6)$ & $52.5(46.1)$ & $47.5(28.3)$ & $45.8(31.9)$ & $46.1(30.7)$ \\
\hline Pseudomonas aeruginosa & 69.3 & 69.9 & 85.0 & 84.7 & 100 & $66.2(75.0)$ & $60.5(51.2)$ & $48.3(24.3)$ & $44.8(31.6)$ & $47.0(28.3)$ \\
\hline Acinetobacter anitratum & 71.3 & 70.5 & 84.5 & 83.2 & 87.3 & 100 & $55.6(50.5)$ & $49.9(31.0)$ & $47.7(32.7)$ & $48.9(25.7)$ \\
\hline Corynebacterium glutamicum & 74.1 & 75.3 & 69.1 & 67.6 & 75.3 & 69.0 & 100 & $47.0(30.2)$ & $47.1(34.3)$ & $49.3(25.9)$ \\
\hline Mycobacterium smegmatis & 74.1 & 76.0 & 68.6 & 67.6 & 69.6 & 68.7 & 89.0 & 100 & $46.4(29.2)$ & $51.0(31.2)$ \\
\hline Bacillus coagulans & 51.4 & 71.1 & 77.3 & 76.5 & 76.9 & 76.9 & 75.2 & 61.6 & 100 & $48.2(28.4)$ \\
\hline Thermoplasma acidophilum & 59.6 & 58.9 & 59.2 & 56.0 & 58.4 & 58.5 & 49.5 & 49.4 & 57.9 & 100 \\
\hline
\end{tabular}

${ }^{a}$ The values on the upper right are the levels of similarity between $g l t A$ sequences and (in parentheses) between citrate synthase amino acid sequences, and the values on the lower left are the levels of similarity between $16 \mathrm{~S}$ rDNA sequences.

class of the Proteobacteria, the representatives of the $\gamma$ subclass of the Proteobacteria, and the representatives of the grampositive bacteria. However, the organization of these clusters was different. The bootstrap values for all of the nodes provided high levels of confidence in the two analyses for the most part; the two exceptions were the branching of Coxiella burnetii $(62 \%)$ on the dendrogram inferred from citrate synthase gene data and the branching of Bacillus coagulans (75\%) on the $16 \mathrm{~S}$ rRNA gene dendrogram.

\section{DISCUSSION}

Currently, serological typing by microimmunofluorescence with mouse polyclonal antisera remains the reference method for identification of rickettsiae, and determination of a new species is officially based on the specificity difference values calculated from microimmunofluorescence results (35). The antigenic determinants for this serotyping are two high-molecular-weight outer membrane proteins, rOmpA and rOmpB, for the SFG rickettsiae and rOmpB for the TG rickettsiae. This method, which requires culture of all recognized serotypes and production of antibodies in laboratory animals, is time-consuming, expensive, and sometimes hazardous. It was proposed almost 20 years ago because only phenotypic criteria were available at the time. Today, it is evident that a polyphasic approach, in keeping with the approaches used for other organisms, would be more suitable. Relatedness by DNA-DNA hybridization, the "gold standard" for bacterial species definition (52), has been used to study relationships between $R$. rickettsii and other species, including $R$. conorii (levels of DNA-DNA hybridization, 91 to $94 \%), R$. sibirica (70 to $74 \%$ ), $R$. montana $(73 \%)$, R. australis $(53 \%)$, R. akari $(46 \%)$, R. prowazekii $(47 \%)$, $R$. typhi $(42 \%)$, and $R$. canada $(47 \%)$. If "the $70 \%$ relatedness rule" were followed, $R$. rickettsii, $R$. conorii, $R$. sibirica, and $R$. montana would belong to the same species (51). However, $R$. rickettsii would be considered a species different from $R$. akari and $R$. australis, two members of the SFG, and would be considered different from $R$. prowazekii, $R$. typhi, and $R$. can$a d a$, three representatives of the TG $(27,51)$. On the basis of this criterion, $R$. prowazekii and $R$. typhi (level of relatedness as determined by DNA-DNA hybridization, $72 \%$ ) would belong to the same species, but $R$. canada would be considered a different species than $R$. prowazekii (45\%) and R. typhi (42\%) (27). Defining rickettsial species by using this criterion, is, however, problematic. First, culture of these bacteria is difficult and time-consuming; thus, using this method each time that a new strain is isolated is almost impossible. Second, interspecies DNA-DNA homology assessments were first made with members of the Enterobacteriaceae, and the critical value of $70 \%$ was proposed based on the results obtained for representatives of this family. The genome of the rickettsiae is four times smaller than the genomes of enteric bacteria (1.0 to $1.4 \mathrm{Mb}$ for most of the rickettsiae and $4.8 \mathrm{Mb}$ for $E$. coli). It has been hypothesized that the obligately intracellular bacteria evolved from free-living bacteria which lost genes which became unnecessary (53). Therefore, most likely, the compositions of the genomes of these bacterial species are more similar. Thus, the observations established by using members of the Enterobacteriaceae are not necessarily applicable to the rickettsiae.

The introduction of molecular techniques has revolutionized the study of gene and genome evolution and has allowed new approaches to phylogenetic inference. In particular, molecular sequences are useful for comparisons between distantly related species or when biochemical characteristics have proven to be inadequate. Recently, a universal phylogenetic study of a vast spectrum of organisms was performed in which $16 \mathrm{~S}$ rDNA sequences were compared, and this study defined three domains, Archaea, Eukarya, and Bacteria (61). The domain Bacteria presently comprises more than 11 phyla, some of which (Proteobacteria, gram-positive bacteria) are subdivided. The rickettsiae are located in group 1 of the $\alpha$ subclass of the phylum Proteobacteria. The order Rickettsiales as described in Bergey's Manual of Systematic Bacteriology has been shown to be phylogenetically diverse. Coxiella burnetii, the agent of $\mathrm{Q}$ fever, has now been removed from the order as it has been shown to be evolutionarily distinct, and to be a member of the $\gamma$ subclass of the Proteobacteria (54). Moreover, the genus Rochalimaea was united with the genus Bartonella in group 2 of the $\alpha$ subclass of the Proteobacteria and was also removed from the Rickettsiales on the basis of significant taxonomic differences, including phylogenetic diversity (10). 16S rRNA-based phylogeny has also been used to infer unexpected evolutionary relationships within the order Rickettsiales. Members of the genus Ehrlichia have been found to form two clearly distinct 

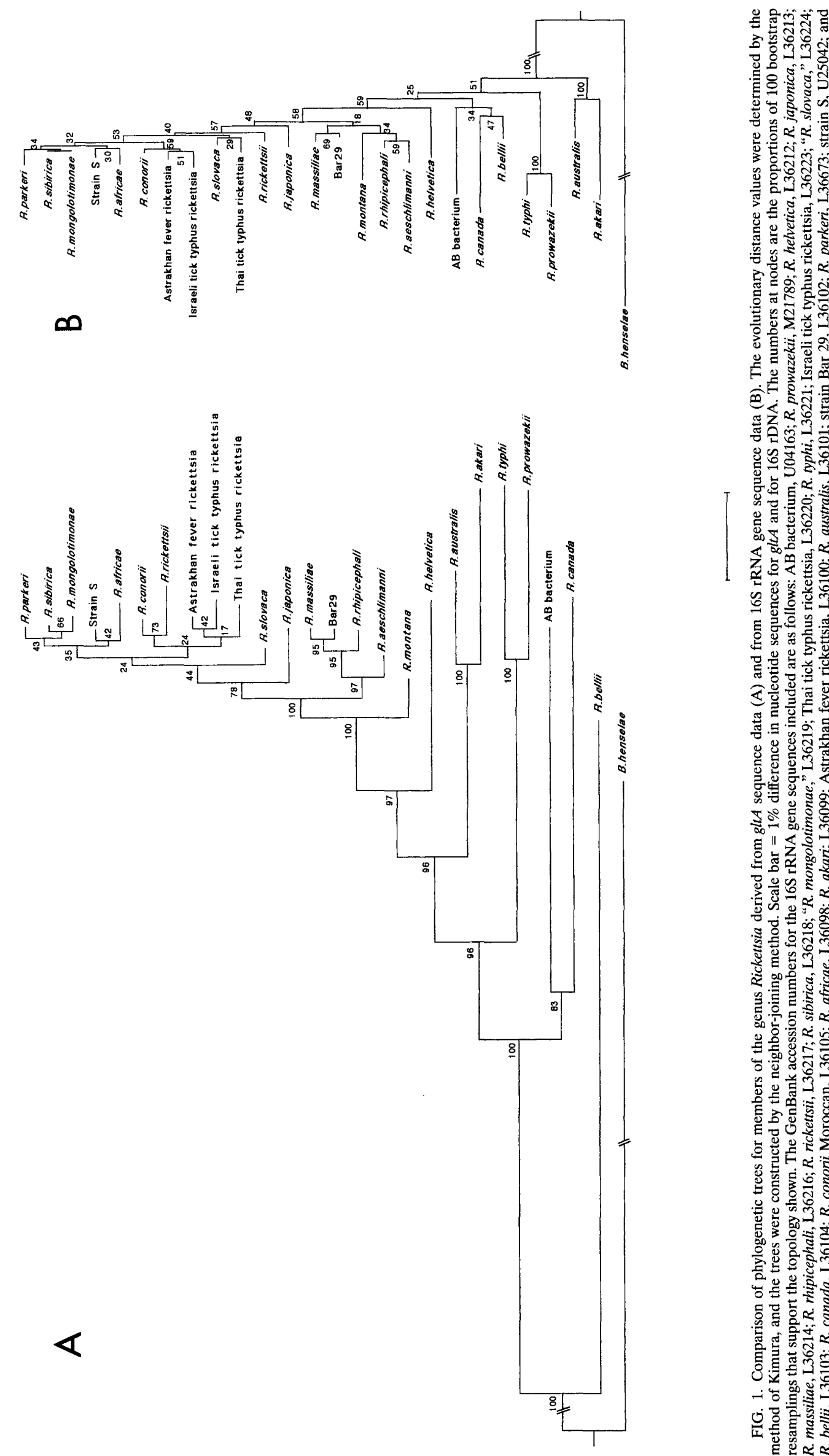

थै क्षे

结要

总虫

品 论

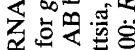

出论

헝 矛运

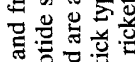

过递要这

政

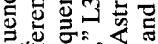

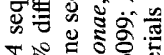

क⿺辶一

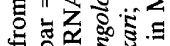

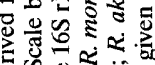

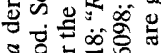

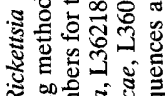

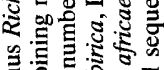

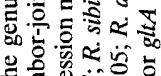

解记

造语

它论

过

昰言客

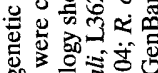

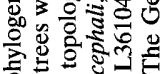

范范

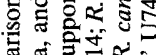

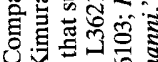

-4 .

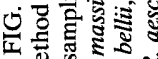



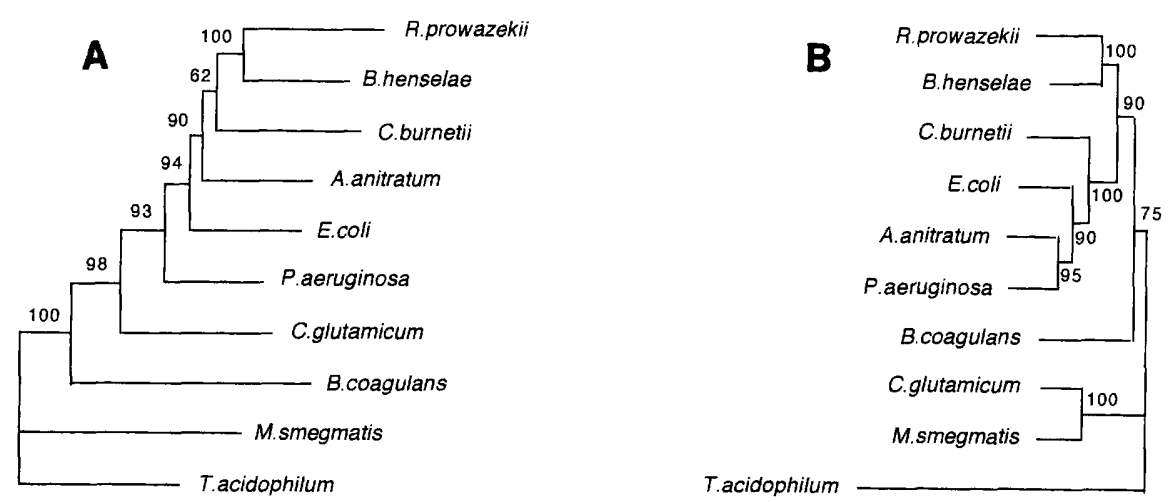

FIG. 2. Distance matrix trees derived from gltA data (A) and from data for the $16 \mathrm{~S}$ rRNA-encoding gene (B). The evolutionary distances were determined by the method of Kimura. These values were used to construct a dendrogram by the neighbor-joining method. The numbers at nodes are the proportions of 100 bootstrap resamplings that support the topology shown. Scale bar $=18 \%$ difference in nucleotide sequences. The GenBank accession numbers for the glt $A$ sequences included are as follows: $R$. prowazekii, M17149; Bartonella henselae, L38987; Coxiella burnetii, M36338; Acinetobacter anitratum, M33037; E. coli, J01619; P. aeniginosa, M29728 Corynebacterium glutamicum, X66112; Bacillus coagulans, M74818; M. smegmatis, X60513; and Thermoplasma acidophilum, X55282. The GenBank accession numbers for the 16S rRNA gene sequences of these bacteria are M21789, M73229, M21291, U10874, M24996, X06684, X80629, D16267, X52922, and M38637, respectively.

lineages (2). Furthermore, $R$. tsutsugamushi has been shown to exhibit only $90 \%$ sequence similarity with other members of this genus, which resulted in a proposal that it should be transferred to the new genus Orientia (48). However, the usefulness of the $16 \mathrm{~S}$ rRNA gene as a tool for evolutionary inference for other members of the genus Rickettsia is less certain. Phylogenetic analyses of members of the SFG or the TG result in models whose branching orders have no statistical significance $(41,45)$. In addition to these problems, shortcomings in overreliance of bacterial phylogeny on comparisons of $16 \mathrm{~S}$ rRNA gene sequences have been highlighted. We therefore investigated the usefulness of an alternative tool for phylogenetic inference in the genus Rickettsia. To date, only a few genes in the rickettsial chromosome have been sequenced, and we chose one of these, the glt $A$ gene, as the gene product is an enzyme central to the metabolism of nearly all living cells (56).

Comparisons of similarity values showed that this gene is less conserved than the $16 \mathrm{~S}$ rRNA gene in rickettsiae. This observation was particularly true for the deepest-branching representatives of the genus Rickettsia. The dendrograms inferred from 16S rDNA and glt $A$ sequences had different architectures in the cluster which included $R$. parkeri, $R$. sibirica, " $R$. mongolotimonae," strain S, $R$. africae, $R$. conorii, $R$. rickettsii, the Astrakhan fever rickettsia, the Israeli tick typhus rickettsia, the Thai tick typhus rickettsia, " $R$. slovaca," and $R$. japonica. The phylogenetic positions of $R$. rickettsii and the Thai tick typhus rickettsia were different; however, in both trees there was no statistical support for their locations. The problem of the phylogenetic organization within this subgroup therefore remains. For these serotypes an additional more rapidly evolving housekeeping molecule is required as an alternative phylogenetic tool. Perhaps the nucleotide or protein sequences of proteins rOmpA and $\mathrm{rOmpB}$, which are specific for the SFG rickettsiae and the members of the genus Rickettsia, respectively, may be suitable candidates. These molecules have been shown to exhibit higher degrees of difference between different strains with PCR-RFLP $(18,38,39)$ or sequencing (39) than other molecules (citrate synthase, $17-\mathrm{kDa}$ protein, 16S rRNA). Differences in the phylogenies inferred from gltA and $16 \mathrm{~S}$ rDNA sequences were found in the organization of the bacteria which represented the most outlying rickettsiae ( $R$. bellii, $R$. canada, the $\mathrm{AB}$ bacterium, $R$. prowazekii, $R$. typhi, $R$. australis, $R$. akari) (Fig. 1). Although $R$. canada and $R$. bellii have some characteristics of the SFG (serological cross-reactions, ticks are the arthropod reservoir, transovarial transmission, and growth in the cytoplasm and nucleus of the host cell), they also have some of the characteristics associated with the TG (identical $\mathrm{G}+\mathrm{C}$ contents) $(27,34)$. Furthermore, the similarities in the sodium dodecylsulfate-polyacrylamide gel electrophoresis patterns of the TG and SFG strains were greater than the similarities between either group and $R$. bellii (34). The AB bacterium was assigned to the genus Rickettsia on the basis of the results of a comparison of $16 \mathrm{~S}$ rDNA and glt $A$ sequences and the results of PCR amplification of parts of the genes encoding two specific rickettsial proteins $(17-\mathrm{kDa}$ protein and rOmpB $)(4,57)$. Characterization of this strain has not yet been completed because cell culture is not possible yet. $R$. canada, $R$. bellii, and the AB bacterium probably represent three new groups distinct from the SFG or the TG. With both methods of analysis $R$. prowazekii and $R$. typh $i$ clustered on the same branch with high bootstrap values $(100 \%)$. This finding is consistent with other phenotypic and genotypic characteristics. These two bacteria are without doubt two representatives of the TG.

$R$. akari and $R$. australis clustered together, and it is reasonable to consider them outlying members of SFG on the basis of the following characteristics: presence of ompA in the R. akari genome (20), serological cross-reactions, and $\mathrm{G}+\mathrm{C}$ contents and genome sizes identical to those of other representatives of the SFG $(40,55)$. The phylogenetic position of $R$. helvetica and the existence of a subgroup that includes $R$. massiliae, strain Bar $29, R$. rhipicephali, $R$. montana, and " $R$. aeschlimanni" were confirmed statistically by the glt $A$ analysis. Recently, Birtles and Raoult (8) showed that the intra-Bartonella architecture of trees inferred from a glt $A$ sequence comparison included four well-supported lineages within the genus, whereas the trees inferred from 16S rDNA sequences had no statistical support. The levels of similarity between Bartonella glt $A$ sequences were markedly lower than the levels of similarity between $16 \mathrm{~S}$ rDNA sequences (8). The average rate of sequence change in glt $A$ is faster than the average rate of sequence change in 16S rRNA; 
thus, for close relationships the glt $A$ sequences can be quite valuable.

We wanted to test the glt $A$ sequence comparison approach for general phylogenic inferences. We obtained from GenBank the glt $A$ sequences available for representatives of the Bacteria and compared the phylogenetic inferences made for these bacteria on the basis of the glt $A$ and $16 \mathrm{~S}$ rDNA data. The phylogenies inferred from the two approaches were similar. We observed that the species assigned to the $\alpha$ and $\gamma$ proteobacterial subclasses by the rRNA analysis also clustered in glt $A$ trees. The species included in the gram-positive bacterial cluster based on 16S rDNA sequences also clustered together when glt $A$ sequences were compared. However, the levels of similarity between the Corynebacterium glutamicum glt $A$ sequence and the gltA sequences of members of the Proteobacteria (60.5 to $51.2 \%$ ) were higher than the levels of similarity obtained for two other members of the gram-positive bacterial cluster, $M$. smegmatis and Bacillus coagulans (49.9 to $44.1 \%$ ). This difference is not consistent with segregation of these organisms into high- and low-G+C-content groups, as Corynebacterium glutamicum and $M$. smegmatis cluster with the high-G+C-content gram-positive bacteria and Bacillus coagulans clusters with the low-G+C-content gram-positive bacteria. On the basis of citrate synthase amino acid sequences, Sutherland et al. concluded that the Eukarya, Bacteria, and Archaea are three separate groups (47). However, no gram-positive bacterial sequence was included in this study. We showed that the division of the Archaea and Bacteria is not so evident on the basis of citrate synthase amino acid and glt $A$ sequence comparisons. In this case, the organization of gram-positive bacteria and the low levels of similarity within this phylum might be apparent only because of the peculiar story of the citrate synthase gene. More glt $A$ sequences will be necessary to study the evolution of representatives of the domain Bacteria (particularly other phyla, including the spirochetes, the Planctomyces-Chlamydia subgroup, the Cyanobacteria, and other subclasses of the Proteobacteria) and to determine the utility of the technique. We found unexpected results for the gram-positive bacterial phylum which need to be confirmed and explained.

In rickettsial phylogeny, although $16 \mathrm{~S}$ rDNA data must be considered not useful, data derived from comparisons of $g l t A$ sequences are consistent with other phenotypic and genotypic characteristics of these organisms. The glt $A$ sequence analysis allowed us to determine more reliable evolutionary relationships within the genus Rickettsia. Based on the calibration of rRNA gene evolution by Ochman and Wilson (31), the divergence of the genera Orientia and Rickettsia occurred $500 \times 10^{6}$ years ago, and divergence among the members of the genus Rickettsia occurred more recently. At present, based on results obtained for Bartonella species and Rickettsia serotypes, phylogenetic studies based on comparisons of glt $A$ sequences should be considered an interesting approach which should be considered when phylogenetic relationships between taxa cannot be significantly resolved by $16 \mathrm{~S}$ rDNA data, particularly when the phylogenetic organization of bacteria whose divergence is as recent as that of members of the genus Rickettsia is studied.

\section{ACKNOWLEDGMENTS}

We thank Richard Birtles for reviewing the manuscript.

This research was made possible in part by grant M2800 from the International Science Foundation.

\section{REFERENCES}

1. Anacker, R. L., R. E. Mann, and C. Gonzales. 1987 . Reactivity of monoclonal antibodies of Rickettsia rickettsii with the spotted fever and typhus group rickettsiae J. Clin Microbiol, 25:167-171.

2. Anderson, B. E., J. E. Dawson, D. C. Jones, and K. H. Wilson. 1991. Ehrlichia chaffeensis, a new species associated with human ehrlichiosis. J. Clin. Microbiol. 29:2838-2842.

3. Babalis, T Y. Tselentis, V. Roux, A. Psaaroulaki, and D. Raoult. 1994 Isolation and identification of a rickettsial strain related to Rickettsia massiliae in Greek ticks. Am. J. Trop. Med. Hyg. 50:365-372.

4. Balayeva, N. M., M. E. Eremeeva, H. Tissot-Dupont, I. A. Zakharov, and D. Raoult. 1995. Genotype characterization of the bacterium expressing the male-killing trait in the ladybird beetle Adalia bipunctata with specific rickettsial molecular tools. Appl. Environ. Microbiol. 61:1431-1437.

5. Beati, L., M. Meskini, and D. Raoult. Rickettsia aeschlimanni: a new spotted fever group rickettsia associated with Hyalomma marginatum ticks. Submitted for publication.

6. Beati, L., J.-P. Finidori, B. Gilot, and D. Raoult. 1992. Comparison of serologic typing, sodium dodecyl sulfate-polyacrylamide gel electrophoresis protein analysis, and genetic restriction fragment length polymorphism analysis for identification of rickettsiae: characterization of two new rickettsial strains. J. Clin. Microbiol. 30:1922-1930.

7. Beati, L., V. Roux, A. Ortuno, J. Castella, F. Segura-Porta, and D. Raoult. 1996. Characterization of spotted fever group rickettsiae isolated from Catalan Rhipicephalus sanguineus ticks. J. Clin. Microbiol. 34:2688-2694.

8. Birtles, R. J., and D. Raoult. 1996. Comparison of partial citrate synthase gene ( $g l t A)$ sequences for phylogenetic analysis of Bartonella species. Int. $\mathrm{J}$. Syst. Bacteriol. 46:891-897.

9. Bloxham, D. P., D. C. Parmelee, S. Kumar, K. A. Walsh, and K. Titani. 1982. Complete amino acid sequence of porcine heart citrate synthase. Biochemistry 21:2028-2036.

10. Brenner, D. J, S. P. O'Connor, H. H. Winkler, and A. G. Steigerwalt. 1993 Proposals to unify the genera Bartonella and Rochalimaea, with descriptions of Bartonella quintana comb. nov., Bartonella vinsonii comb. nov., Bartonella henselae comb. nov., and Bartonella elizabethae comb. nov., and to remove family Bartonellaceae from the order Rickettsiales. Int. J. Syst. Bacteriol. 43:777-786.

11. Burgdorfer, W., A. Aeschlimann, O. Peter, S. F. Hayes, and R. N. Philip. 1979. Ixodes ricinus: vector of a hitherto undescribed spotted fever group agent in Switzerland. Acta Trop. 39:357-367.

12. David, M., S. Lubinsky-Mink, A. Ben-Zui, M. Suissa, S. Unitzur, and J. Kuhn. 1991. Citrate synthase from Mycobacterium smegmatis. Biochem. J. 278:225-234.

13. Dessen, P., C. Fondrat, C. Valencien, and G. Munier. 1990. BISANCE: a French service for access to biomolecular sequences databases. CABIOS 6:355-356.

14. Donald, L. J., and H. W. Duckworth. 1987. Expression and base sequence of the citrate synthase gene of Acinetobacter anitratum. Biochem. Cell Biol. 65:930-938.

15. Donald, L. J., G. F. Molgat, and H. W. Duckworth. 1989. Cloning, sequencing, and expression of the gene for NADH-sensitive citrate synthase of Pseudomonas aeruginosa. J. Bacteriol. 171:5542-5550.

16. Eikmanns, B. J., N. Thum-Schmitz, L. Eggeling, K. U. Luedtke, and H. Sahm. 1994. Nucleotide sequence, expression and transcriptional analysis of Corynebacterium glutamicum glt A gene encoding citrate synthase. Microbiology 140:1817-1828.

17. Eremeeva, M., L. Beati, V. A. Makarova, N. F. Fetisova, I. V. Tarasevitch, N. M. Balayeva, and D. Raoult. 1994. Astrakhan fever rickettsiae: antigenic and genotypic analysis of isolates obtained from human and Rhipicephalus pumilio ticks. Am. J. Trop. Med. Hyg. 51:697-706.

18. Eremeeva, M., X. Yu, and D. Raoult. 1994. Differentiation among spotted fever group rickettsia species by analysis of restriction fragment length polymorphism of PCR-amplified DNA. J. Clin. Microbiol. 32:803-810

19. Felsenstein, J. 1989. PHYLIP-phylogeny inference package (version 3.2) Cladistics 5:164-166.

20. Gilmore, R. D., Jr. 1993. Comparison of the rOmpA gene repeat regions of rickettsiae reveals species-specific arrangements of individual repeating units. Gene 125:97-102.

21. Heinzen, R. A., M. E. Frazier, and L. P. Mallavia. 1991. Sequence and linkage analysis of the Coxiella bumetii citrate synthase-encoding gene. Gene 109:63-69.

22. Higgins, J. A., S. Radulovic, M. E. Schrieffer, and A. F. Azad. 1996. Rickettsia felis: a new species of pathogenic rickettsia isolated from cat fleas. J. Clin. Microbiol. 34:671-674.

23. Jukes, T. H., and C. R. Cantor. 1969. Evolution of protein molecules, p. 21-132. In H. N. Munro (ed.), Mammalian protein metabolism, vol. 3 Academic Press, Inc., New York, N.Y.

24. Kelly, P., L. Matthewman, L. Beati, D. Raoult, P. Mason, M. Deary, and R. Makombe. 1992. African tick-bite fever: a new spotted fever group rickettsiosis under an old name. Lancet 340:382-383.

25. Kimura, M. 1980 . A simple method for estimating evolutionary rates of base substitutions through comparative studies of nucleotide sequences. J. Mol Evol. 16:111-120

26. Marrero, M., and D. Raoult. 1989. Centrifugation shell vial technique for 
rapid detection of mediterranean spotted fever in blood culture. Am. J. Trop. Med. Hyg. 40:197-199.

27. Myers, W. F., and C. L. Wisserman, Jr. 1981. The taxonomic relationship of Rickettsia canada to the typhus and spotted fever groups of the genus Rick ettsia, p. 313-325. In W. Burgdorfer and L. R. Anacker (ed.), Rickettsiae and rickettsial diseases. Academic Press, New York, N.Y.

28. Ner, S. S., V. Bhayana, A. W. Bell, I. G. Giles, H. W. Duckworth, and D. P. Bloxham. 1983. Complete sequence of the glt A gene encoding citrate synthase in Escherichia coli. Biochemistry 22:5243-5249.

29. Norman, A. F., R. Regnery, P. Jameson, C. Greene, and D. C. Krause. 1995 Differentiation of Bartonella-like isolates at the species level by PCR-restriction fragment length polymorphism in the citrate synthase gene. J. Clin. Microbiol. 33:1797-1803.

30. Numata, O., T. Takemasa, I. Takagi, M. Hirono, H. Hirano, and Y. Watanabe. 1991. Tetrahymena 14-nm filament-forming protein has citrate synthase activity. Biochem. Biophys. Res. Commun. 174:1028-1034.

31. Ochman, H., and A. C. Wilson. 1987. Evolution in bacteria: evidence for a universal substitution rate in cellular genomes. J. Mol. Evol. 26:74-86.

32. Oisen, G. J., and C. R. Woese. 1993. Ribosomal RNA: a key to phylogeny FASEB J. 7:113-123.

33. Phibbs, P. V., Jr., and H. H. Winkler. 1982. Regulatory properties of citrate synthase from Rickettsia prowazekii. J. Bacteriol. 149:718-725.

34. Philip, R. N., E. A. Casper, R. L. Anacker, J. Cory, S. F. Hayes, W. Burg dorfer, and C. E. Yunker. 1983. Rickettsia bellii sp. nov.: tick-borne rickettsia, widely distributed in the United States, that is distinct from the spotted fever and typhus biogroups. Int. J. Syst. Bacteriol. 33:94-106.

35. Philip, R. N., E. A. Casper, W. Burgdorfer, R. K. Gerloff, L. E. Hughes, and E. J. Bell. 1978. Serologic typing of rickettsiae of spotted fever group by microimmunofluorescence. J. Immunol. 121:1961-1968.

36. Ralph, D., C. Pretzman, N. Daugherty, and K. Poetter. 1990. Genetic relationships among the members of the family Rickettsiaceae as shown by DNA restriction fragment polymorphism analysis. Ann. N.Y. Acad. Sci. 590:541553.

37. Raoult, D., P. Brouqui, and V. Roux. 1996. A new spotted fever group rickettsiosis. Lancet 348:412.

38. Regnery, R. L., C. L. Spruill, and B. D. Plikaytis. 1991. Genotypic identification of rickettsiae and estimation of intraspecies sequence divergence for portions of two rickettsial genes. J. Bacteriol. 173:1576-1589.

39. Roux, V., P. E. Fournier, and D. Raoult. 1996. Identification of the spotted fever group rickettsiae by sequencing and analysis of restriction fragment length polymorphism of PCR-amplified DNA of the gene encoding the protein rOmpA. J. Clin. Microbiol. 34:2058-2065.

40. Roux, V., and D. Raoult. 1993. Genotypic identification and phylogenetic analysis of the spotted fever group rickettsiae by pulsed-field gel electrophoresis. J. Bacteriol. 175:4895-4904.

41. Roux, V., and D. Raoult. 1995. Phylogenetic analysis of the genus Rickettsia by 16S rDNA sequencing. Res. Microbiol. 146:385-396.

42. Saitou, $\mathbf{N}_{\text {, }}$ and M. Nei. 1987. The neighbor-joining method: a new method for reconstructing phylogenic trees. Mol. Biol. Evol. 4:406-425.

43. Schendel, F. J., P. R. August, C. R. Anderson, R. S. Hanson, and M. C Flickinger. 1992. Cloning and nucleotide sequence of the gene coding for citrate synthase from a thermotolerant Bacillus sp. Appl. Environ. Microbiol. 58:335-345.

44. Schriefer, M. E J. B. Sacci, Jr., S. Dumler, M. G. Bullen, and A. F. Azad. 1994. Identification of a novel rickettsial infection in a patient diagnosed with murine typhus. J. Clin Microbiol. 32:949-954

45. Stothard, D. R., and P. A. Fuerst. 1995. Evolutionary analysis of the spotted fever and typhus groups of Rickettsia using 16S rRNA gene sequences. Syst. Appl. Microbiol. 18:52-61.

46. Suissa, M., K. Suda, and G. Schatz. 1984. Isolation of the nuclear yeast genes for citrate synthase and fifteen other mitochondrial proteins by a new screening method. EMBO J. 3:1773-1781.

47. Sutherland, K. J., C. M. Henneke, P. Towner, D. W. Hough, and M. J. Danson. 1990. Citrate synthase from the thermophilic archaebacterium Ther moplasma acidophilum, cloning and sequencing of the gene. Eur. J. Biochem 194:839-844.

48. Tamura, A., N. Ohashi, H. Urakami, and S. Miyamura. 1995. Classification of Rickettsia tsutsugamushi in a new genus, Orientia gen. nov., as Orientia tsutsugamushi comb. nov. Int. J. Syst. Bacteriol. 45:589-591.

49. Uchida, T., T. Uchiyama, K. Kumano, and D. H. Walker. 1992. Rickettsio japonica sp. nov., the etiological agent of spotted fever group rickettsiosis in Japan. Int. J. Syst. Bacteriol 42:303-305.

50. Unger, E. A., J. M. Hand, A. R. Cashmore, and A. C. Vasconcelos. 1989 Isolation of a cDNA encoding mitochondrial citrate synthase from Arabidopsis thaliana. Plant Mol. Biol. 13:411-418.

51. Walker, D. H. 1989. Rocky mountain spotted fever: a disease in need of microbiological concern. Clin. Microbiol. Rev. 2:227-240.

52. Wayne, L. G., D. J. Brenner, R. R. Colwell, P. A. D. Grimont, O. Kandler, M. I. Krichevsky, L. H. Moore, W. E. C. Moore, R. G. E. Murray, E. Stackebrandt, M. P. Starr, and H. G. Trûper. 1987. Report of the Ad Hoc Committee on Reconciliation of Approaches to Bacterial Systematics. Int. J. Syst. Bacteriol. 37:463-464.

53. Weisburg, W. G. 1985. Polyphyletic origin of bacterial parasites, p. 1-15. In J. W. Moulder (ed.), Intracellular parasitism. CRC Press, Inc., Boca Raton, Fla.

54. Weisburg, W. G., M. E. Dobson, J. E. Samuel, G. A. Dasch, L. P. Mallavia, O. Baca, L. Mandelco, J. E. Sechrest, E. Weiss, and C. R. Woese, 1989. Phylogenetic diversity of rickettsiae. J. Bacteriol. 171:4202-4206.

55. Weiss, E., and J. W. Moulder. 1984. Order I. Rickettsiales Gieszczkiewicz $1939,25^{\mathrm{AL}}$, p. $687-701$. In N. R. Krieg and J. G. Holt (ed.), Bergey's manual of systematic bacteriology, vol. 1. The Williams and Wilkins Co., Baltimore, $\mathrm{Md}$

56. Weitzman, P. D. J., and M. J. Danson. 1976. Citrate synthase. Curr. Top. Cell. Regul. 10:161-204.

57. Werren, J. H., G. D. D. Hurst, W. Zhang, J. A. J. Breeuvwer, R. Stouthamer, and M. E. N. Majerus. 1994. Rickettsial relative associated with male killing in the ladybird beetle (Adalia bipunctata). J. Bacteriol. 176:388-394.

58. Wiegand, G., and S. J. Remington. 1986. Citrate synthase: structure, control, and mechanism. Annu. Rev. Biophys. Biophys. Chem. 15:97-117.

59. Winkler, H. H. 1976. Rickettsial permability. J. Mol. Chem. 251:389-396

60. Woese, C. R. 1987. Bacterial evolution. Microbiol. Rev. 51:221-271.

61. Woese, C. R., O. Kandler, and M. Wheelis. 1990. Towards a natural system of organisms: proposal for the domains Archaea, Bacteria, and Eukarya. Proc. Natl. Acad. Sci. USA 87:4576-4579.

62. Wood, D. O, L. R. Williamson, H. H. Winkler, and D. C. Krause. 1987. Nucleotide sequence of the Rickettsia prowazekii citrate synthase gene. J. Bacteriol. 169:3564-3572.

63. Yu, X., Y. Jin, M. Fan, G. Xu, Q. Liu, and D. Raoult. 1993. Genotypic and antigenic identification of two new strains of spotted fever group rickettsiae isolated from China. J. Clin. Microbiol. 31:83-88. 